Tendências em Matemática Aplicada e Computacional, 3, No. 2 (2002), 63-72.

(C) Uma Publicação da Sociedade Brasileira de Matemática Aplicada e Computacional.

\title{
Um Esquema GMRES Precondicionado para Simulação de Reservatórios
}

L.M. CARVALHO ${ }^{1}$, Departamento de Matemática Aplicada, Instituto de Matemática e Estatística, UERJ, Rua São Francisco Xavier 524, 20550-013, Rio de Janeiro, RJ, Brasil

F. DICKSTEIN², Departamento de Matemática Aplicada, Instituto de Matemática, UFRJ, Avenida Brigadeiro Trompowski s/n, 21945-970, Rio de Janeiro, RJ, Brasil

J.R.P. RODRIGUES 3 , PETROBRAS/CENPES/PDEP/TR, Cidade Universitária, QD 7, 21949-900, Rio de Janeiro, RJ, Brasil

R.W. dos SANTOS ${ }^{4}$, Instituto de Matemática, UFRJ, Avenida Brigadeiro Trompowski s/n, 21945-970 Rio de Janeiro, RJ, Brasil.

Resumo. Descrevemos um método GMRES precondicionado para a resolução de sistemas lineares que aparecem em Simulação de Reservatórios de Petróleo. Três esquemas de precondicionamento são propostos. Resultados numéricos e uma comparação com um simulador comercial são apresentados. Em particular, uma aplicação a um problema de determinação de parâmetros é discutida.

\section{Introdução}

Neste trabalho descrevemos um esquema iterativo do tipo gradientes conjugados generalizados para a resolução de sistemas lineares, especializado para um tipo particular de aplicação, o da Simulação de Reservatórios de Petróleo. Este esquema foi implementado no pacote computacional COMBESP, trabalho desenvolvido dentro de um projeto CTPETRO que envolveu a Universidade Federal do Rio de Janeiro, a Universidade do Estado do Rio de Janeiro e o Centro de Pesquisas da Petrobrás. O interesse para a Petrobrás é o de possuir um pacote de rotinas eficientes e sobre o qual ela tenha amplo domínio e conhecimento, de forma a poder utilizá-lo e, eventualmente, modificá-lo para o uso em certas aplicações particulares. Por exemplo,

\footnotetext{
${ }^{1}$ luizmc@ime.uerj.br, esse autor foi parcialmente financiado pela FINEP, através do projeto CTPETRO-951.

${ }^{2}$ flavio@labma.ufrj.br

${ }^{3}$ zeroberto@cenpes.petrobras.com.br, Departamento de Análise Matemática, Instituto de Matemática e Estatística, UERJ.

${ }^{4}$ rodrigo@labma.ufrj.br
} 
para fazer o chamado ajuste histórico, que discutiremos adiante. Não descreveremos aqui as diversas características do COMBESP, nem as opções que o pacote apresenta. Tais informações podem ser encontradas em [3].

Do ponto de vista matemático, deseja-se resolver numericamente um sistema de equações diferenciais que modelam o fluxo multifásico em um meio poroso. Este é um problema importante e altamente complexo, e um enorme esforço tem sido feito nas últimas décadas na busca do entendimento dos mecanismos centrais que governam o fenômeno, e na procura de métodos numéricos eficientes para a sua simulação. O leitor poderá encontrar em $[2,15]$, e em suas referências, os elementos para retraçar esta história. Está fora do propósito deste artigo uma descrição dos modelos que são utilizados em Simulação de Reservatórios de Petróleo ou dos esquemas numéricos usualmente empregados. No entanto, na Seção 2, apresentamos algumas características básicas do sistema para que o leitor possa compreender como são obtidos os sistemas lineares que iremos resolver. Na Seção 3, mostramos como o chamado ajuste histórico se traduz em um problema de determinação de parâmetros, que por sua vez conduz à resolução de um sistema linear de múltiplos lados direitos. A seguir, descrevemos, na Seção 4, o COMBESP, esquema iterativo por nós desenvolvido, enquanto que na Seção 5 apresentamos um algoritmo para a resolução de múltiplos lados direitos. Finalmente, na Seção 6, são discutidos alguns resultados numéricos, perspectivas e conclusões.

\section{O Fluxo Bifásico em um Meio Poroso}

Apresentamos a seguir um modelo simples (mas típico) que descreve o processo de injeção de um líquido (água) através de poços injetores, para a recuperação do óleo através de poços produtores. Para facilitar a exposição, e sem prejuízo das idéias centrais que queremos ressaltar, tomamos certas funções espaciais como constantes e iguais a 1, de forma que o modelo consiste em determinar as saturações da água $s_{a}$ e do óleo $s_{o}$ e as pressões da água $p_{a}$ e do óleo $p_{o}$ satisfazendo

$$
\begin{gathered}
\frac{\partial s_{a}}{\partial t}-\nabla \cdot\left(\lambda_{a} \nabla p_{a}\right)=q_{a}, \\
\frac{\partial s_{o}}{\partial t}-\nabla \cdot\left(\lambda_{o} \nabla p_{o}\right)=q_{o} .
\end{gathered}
$$

Aqui, as permeabilidades $\lambda_{a}$ e $\lambda_{o}$ são funções conhecidas das saturações e $q_{a}, q_{o}$ são termos fonte devidos aos poços. Observe que temos quatro incógnitas e duas equações. O sistema é completado usando-se as relações algébricas $s_{a}+s_{o}=1 \mathrm{e}$ $p_{a}-p_{o}=p_{c}$, onde a chamada pressão capilar, $p_{c}$, é também uma função conhecida das saturações. Suponhamos (ainda sem maiores prejuízos para a apresentação das idéias) que $p_{c}=0$. Fazendo $s=s_{a}$ e $p=p_{a}$, somando a duas equações e usando a relação $s_{a}+s_{o}=1$, obtemos o sistema

$$
\frac{\partial s}{\partial t}-\nabla \cdot\left(\lambda_{a}(s) \nabla p\right)=q_{a},
$$




$$
-\nabla \cdot\left(\lambda_{t}(s) \nabla p\right)=q_{t}=q_{a}+q_{o},
$$

onde $\lambda_{t}=\lambda_{a}+\lambda_{o}$. Evidentemente, condições iniciais e de fronteira adicionais devem ser impostas. Para maiores detalhes, ver [2].

Um esquema de diferenças finitas implícito empregado para aproximar (2.1), (2.2) é dado por

$$
\begin{gathered}
\frac{s^{n+1}-s^{n}}{\Delta t}-\nabla_{h}^{d} \cdot\left(\lambda_{a}\left(s^{n+1}\right) \nabla_{h}^{g} p^{n+1}\right)=q_{a}\left(p_{w}^{n+1}\right), \\
-\nabla_{h}^{d} \cdot\left(\lambda_{t}\left(s^{n+1}\right) \nabla_{h}^{g} p^{n+1}\right)=q_{t}\left(p_{w}^{n+1}\right),
\end{gathered}
$$

onde $\Delta t$ é o passo de tempo, $\nabla_{h}^{d}, \nabla_{h}^{g}$ são os operadores divergente e gradiente usuais de diferenças finitas de segunda ordem, respectivamente, e $p_{w}$ indica a pressão nos poços. Cada poço atravessa diversos blocos de discretização do reservatório $b_{i}$, $i=1,2, \ldots, k$, e suas pressões de poço $p_{w}(i)$ e de reservatório $p(i)$ estão relacionadas por equações algébricas do tipo

$$
F\left(p_{w}^{n+1}(1), p_{w}^{n+1}(2), \ldots, p_{w}^{n+1}(k)\right)=0,
$$

veja [2]. (2.3), (2.4), (2.5) formam um sistema não-linear, a ser resolvido em cada instante $n \Delta t$ pelo Método de Newton. Assim, em cada iteração newtoniana devemos resolver um sistema linear algébrico $A x=b$ com a seguinte estrutura

$$
\left(\begin{array}{ccc}
A_{s s} & A_{s p_{r}} & 0 \\
A_{p_{r} s} & A_{p_{r} p_{r}} & A_{p_{r} p_{w}} \\
0 & A_{p_{w} p_{r}} & A_{p_{w} p_{w}}
\end{array}\right)\left(\begin{array}{c}
s \\
p_{r} \\
p_{w}
\end{array}\right)=\left(\begin{array}{c}
b_{s} \\
b_{p_{r}} \\
b_{p_{w}}
\end{array}\right) .
$$

Na próxima seção, discutimos como resolver (2.6).

Observação 2.1 - $\quad$ No caso de fluxos multifásicos e isotérmicos com $j$ componentes, as variáveis a serem resolvidas são: $j-1$ concentrações, a pressão no reservatório e a pressão no poço. O caso bifásico acima corresponde a $j=2$.

\section{O Problema do Ajuste Histórico}

Uma característica importante da Engenharia de Petróleo é a dificuldade de obtenção de dados geológicos para uma boa descrição de um reservatório. Um procedimento consagrado na área é o ajuste histórico, que descrevemos a seguir. Suponha que um reservatório esteja produzindo há algum tempo. A partir do conhecimento de seu histórico de produção, podemos usar um simulador numérico para ajustar os parâmetros do modelo de forma a reproduzir a resposta conhecida.

Portanto, o ajuste histórico consiste no problema inverso de determinar $\mu$ tal que

$$
u(\mu)=\bar{u},
$$

onde

$$
F(u(\mu), \mu)=q .
$$


Aqui, $u=\left(s, p_{r}, p_{w}\right), \mu$ é o vetor de parâmetros a serem determinados (por exemplo, a permeabilidade em cada ponto), $\bar{u}$ é o histórico conhecido, $F$ é o sistema de EDPs discretizado a ser resolvido, $q$ corresponde às vazões nos poços. O método de Newton para resolver (3.1) se escreve como

$$
B\left(\mu^{n+1}-\mu^{n}\right)=q-u\left(\mu^{n}\right),
$$

onde

$$
B=u^{\prime}\left(\mu^{n}\right) .
$$

Derivando (3.2) em relação a $\mu_{i}$, a $i$-ésima componente de $\mu$, obtemos

$$
\frac{\partial F}{\partial u} \frac{\partial u}{\partial \mu_{i}}+\frac{\partial F}{\partial \mu_{i}}=0,
$$

de modo que $u^{\prime}(\mu)$ é obtido resolvendo-se o mesmo sistema linear $n$ vezes, onde $n$ é o tamanho do vetor $\mu$.

\section{O Método Iterativo GMRES Precondicionado}

O sistema (2.3) é resolvido pelo COMBESP por um esquema GMRES (generalized minimum residual) precondicionado. O método GMRES é o esquema de gradientes conjugados generalizados de uso mais difundido em Simulação de Reservatórios, devido à sua eficiência e robustez. O leitor poderá consultar $[6,9]$ para um estudo do GMRES. A técnica de precondicionamento é discutida abaixo.

Um método precondicionado consiste na substituição do sistema linear

$$
A x=b
$$

pelo sistema precondicionado

$$
\tilde{A} x=\tilde{b},
$$

onde $\tilde{A}=M A, \tilde{b}=M b$, sendo $M$ uma matriz inversível. A idéia do precondicionamento é a de obter um sistema equivalente com uma matriz melhor condicionada que a matriz original. Observe que os métodos iterativos usuais requerem o cálculo de multiplicações do tipo $w=\tilde{A} z$, de forma que devemos efetuar $y=A z$ e $w=M y$. Assim, $M$ deverá satisfazer dois requisitos conflitantes. Por um lado, ser próximo de $A^{-1}$ (para que o sistema seja bem condicionado) e tal que $M y$ não seja muito custoso. Para maiores detalhes, veja ainda [5].

O COMBESP utiliza três alternativas de precondicionamento, descritas a seguir.

\subsection{Fatoração incompleta de nível $k_{r}$}

A fatoração incompleta é uma das técnicas de precondicionamento mais populares [1]. Consiste na decomposição $L U$ aproximada, onde desconsideramos preenchimentos provocados pela eliminação gaussiana. Desta forma os fatores $L$ e $U$ têm a estrutura de esparsidade próxima da estrutura de esparsidade da matriz original $A$. Um parâmetro $k_{r}$ indica o nível de enchimento requerido. Iremos denotar a fatoração da forma usual ILU $\left(k_{r}\right)$. Remetemos o leitor a [10] para maiores detalhes. 


\subsection{Combinativo 1}

O esquemas Combinativo foi proposto inicialmente em [14] e corresponde a um precondicionamento da forma

$$
M=G+P-P A G,
$$

onde $G$ e $P$ são dois precondicionadores de $A$. Portanto, $M$ é uma combinação adequada deles dois.

Observação 4.1 - Uma motivação para a introdução de (4.3) é a seguinte. Suponha que $Q$ seja uma matriz $p \times n$ tal que $Q A P=Q$. Então, $Q A M=Q A G+$ $Q A P-Q A P A G=Q$. Suponha agora que o sistema (4.1) seja resolvido por GMRES precondicionado por $M$ como em (4.3), e que com o resíduo inicial $r_{0}$ satisfaça $Q r_{0}=$ 0 . Então, do fato que $Q A M r_{0}=0$ deduz-se que $Q A M r_{n}=0$ para todo $n$, onde $r_{n}$ é o $n$-ésimo resíduo do método iterativo (veja [3]). Dessa forma, GMRES opera em um subespaço complementar ao núcleo de $Q$. (Analogamente, se $G A Q=Q$ então $M A Q=Q$.

Seguiremos [14], tomando $G$ como uma componente global, dada pela fatoração incompleta descrita na Seção 4.1. Para descrever a componente de pressão $P$, vamos decompor (2.3) nas variáveis de pressão e de saturação, o que corresponde a aglutinar as variáveis de pressão no reservatório $p_{r}$ e pressão no poço $p_{w}$ na variável pressão $p$. Desta forma, (2.3) pode ser reescrita como

$$
\left(\begin{array}{ll}
A_{s s} & A_{s p} \\
A_{p s} & A_{p p}
\end{array}\right)\left(\begin{array}{c}
s \\
p
\end{array}\right)=\left(\begin{array}{l}
b_{s} \\
b_{p}
\end{array}\right) .
$$

Então, $P$ é definida por

$$
P=\left(\begin{array}{cc}
0 & 0 \\
0 & A_{P P}^{-1}
\end{array}\right) .
$$

Observação 4.2 - A equação de pressão (2.2) tem um caráter elítico, ao passo que a saturação evolui com um comportamento essencialmente local, veja o seu caráter hiperbólico em (2.1). Desta forma, a necessidade de resolver mais precisamente a variável $p$ justifica a introdução do precondicionamento $P$. Note que, se $Q=(0, I)$ então $Q A P=Q$, e podemos resolver exatamente na variável da pressão, veja observação 4.1 .

Para a inversão de $A_{P P}$, utilizamos novamente um esquema GMRES precondicionado por uma fatoração incompleta de nível $k_{p_{1}}$.

\subsection{Combinativo 2}

Resultados numéricos revelaram que o precondicionamento Combinativo 1 necessita de um número consideravelmente menor de iterações que o precondicionamento 


\begin{tabular}{c|ccc} 
esquema & tot iter & média iter & tempo \\
\hline \hline SIMCOM/ILU(0) & 5681 & 21,1 & 714,7 \\
SIMCOM/ILU(1) & 3723 & 13,8 & 691,2 \\
COMBESP/ILU(1) & 3898 & 14,5 & 868,1 \\
COMB(0,1) & 3445 & 12,8 & 823,7 \\
COMB(0,2) & 3213 & 11,9 & 773,1 \\
COMB(1,1) & 2785 & 10,4 & 806,4 \\
\hline
\end{tabular}

Tabela 1: Comparação do pacote comercial com Combinativo $2\left(\operatorname{Comb}\left(k_{r}, k_{p}\right)\right)$

$\operatorname{ILU}\left(k_{r}\right)$. No entanto, seu custo por interação é bem maior. Isto nos motivou a considerar a esquema Combinativo 2 cuja única diferença em relação ao Combinativo 1 reside no fato de que, ao invés de inverter $A_{P P}$ por um esquema GMRES precondicionado, aproximamos $A_{P P}^{-1}$ por uma fatoração incompleta de nível $k_{p_{2}}$. Tipicamente, $k_{p_{2}}>k_{p_{1}}$. Combinativo 2 mostrou ser o esquema mais eficiente.

\section{O Esquema para Múltiplos Lados Direitos}

Uma aplicação que motivou a elaboração do COMBESP refere-se ao problema do ajuste histórico discutido na Seção 3, que requer a resolução de múltiplos lados direitos. Iremos descrever sucintamente o chamado método de sementes para a resolução de vários lados direitos, introduzido em [7], e que foi implementado no COMBESP. Trata-se de escolher um lado direito, chamado de semente, resolvê-lo através de um esquema de gradientes conjugados (caso simétrico) ou gradientes conjugados generalizados (caso não-simétrico), projetando os resíduos dos outros lados direitos sobre o espaço de Krylov gerado pelo semente. Em seguida, escolhe-se um segundo semente (por exemplo, o de maior resíduo) e repete-se o processo. A justificativa para tal procedimento é a de que a projeção no espaço de Krylov elimina as componentes dos resíduos associadas aos autovalores extremos da matriz, tornando os problemas seguintes melhor condicionados. Os desenvolvimentos teóricos de $[4,8,13]$ fundamentam esta idéia. Para aplicações e exemplos numéricos, veja $[11,12]$.

\section{Resultados Numéricos}

Discutiremos inicialmente o desempenho do COMBESP quando comparado com o de um simulador comercial, que chamaremos aqui de SIMCOM. Reportamos o leitor à Tabela 1, relativa à resolução de um problema trifásico com 9000 células e 26 poços. O critério de parada é: $\|e\|_{2} \leq 10^{-5}$, onde $e$ é o erro relativo. Podemos verificar que o número de iteraçoes para a resolução nos dois pacotes é comparável. O custo temporal por iteração do SIMCOM é cerca de $25 \%$ menor se comparado ao Combinativo 2. 


\begin{tabular}{c|cc} 
lados direitos & ganho tempo & ganho iteração \\
\hline \hline $\mathbf{2}$ & 0,91 & 1,00 \\
$\mathbf{4}$ & 0,74 & 0,90 \\
$\mathbf{8}$ & 0,78 & 0,92 \\
$\mathbf{1 6}$ & 0,63 & 0,93 \\
$\mathbf{3 2}$ & 0,50 & 1,05 \\
$\mathbf{6 4}$ & 0,29 & 1,10 \\
\hline
\end{tabular}

Tabela 2: Esquema de sementes para posto igual ao número de lados direitos

\begin{tabular}{c|cc} 
lados direitos & ganho tempo & ganho iteração \\
\hline \hline $\mathbf{2}$ & 1,67 & 3,50 \\
$\mathbf{4}$ & 1,64 & 2,71 \\
$\mathbf{8}$ & 1,33 & 2,19 \\
$\mathbf{1 6}$ & 0,89 & 2,02 \\
$\mathbf{3 2}$ & 0,60 & 2,06 \\
$\mathbf{6 4}$ & 0,39 & 2,18 \\
\hline
\end{tabular}

Tabela 3: Esquema de sementes para posto igual à metade do número de lados direitos

A propriedade teórica mais relevante do método de sementes é a seguinte. Suponha que os $k$ vetores que constituem o lado direito formem um conjunto de posto $p$. Então, ao final da resolução da p-ésima semente todos os $k$ lados direitos terão sido resolvidos exatamente. Uma forma de depurar erros do programa foi a verificação desta propriedade no nosso caso. As Tabelas 2 e 3 apresentam o ganho (speed up) obtido nos casos em que $p=k$ e em que $p=k / 2$. Observe que no caso em que $p=k / 2$ o ganho no número de iterações tende a se estabilizar em 2, conforme prevê a teoria. No entanto, o custo adicional do esquema semente é bastante alto, em especial porque o problema é não simétrico e exige um esquema de base custoso (em relação ao de gradientes conjugados) como o GMRES. Desta forma, o ganho no tempo decai com o valor de $k$ tornando desinteressante o uso do método a partir de $k=16$, neste exemplo. No caso em que $k=p$, o ganho no número de iterações tende a se estabilizar em 1. Devido ao custo do esquema, o ganho no tempo é menor que 1, de modo que sua aplicação se torna sem interesse. Embora seja apresentado aqui apenas um exemplo, esta foi a situação típica que encontramos. Observemos ainda que para as aplicações que temos em vista (ajuste histórico, determinação do campo de permeabilidades), não podemos esperar nenhum nível de dependência linear entre os lados direitos. Desta forma, as análises desenvolvidas em [4], que indicam que se pode esperar algum ganho na implementação do esquema semente mesmo neste caso, não se verificou para problemas de Simulação de Reservatórios.

Visto que o esquema de sementes não apresentou o desempenho esperado, devemos nos perguntar como proceder para resolver problemas de determinação de parâmetros em Simulação de Reservatórios. A alternativa que se apresenta é a 


\begin{tabular}{c|cccc}
$\mathbf{n}$ & iterações & tempo total & tempo iteração & $k_{r}$ \\
\hline \hline $\mathbf{1}$ & 19 & 5,66 & 5,66 & 1 \\
$\mathbf{1 0}$ & 203 & 33,83 & 3,38 & 1 \\
$\mathbf{2 0}$ & 247 & 67,72 & 3,39 & 3 \\
$\mathbf{3 0}$ & 368 & 90,08 & 3,00 & 3 \\
$\mathbf{4 0}$ & 488 & 125,07 & 3,13 & 3 \\
\hline
\end{tabular}

Tabela 4: Precondicionador ILU(kr)

\begin{tabular}{c|cccc}
$\mathbf{n}$ & iterações & tempo total & tempo iteração & {$\left[k_{r}, k_{p_{1}}\right]$} \\
\hline \hline $\mathbf{1}$ & 5 & 6,15 & 6,15 & {$[1,1]$} \\
$\mathbf{1 0}$ & 59 & 32,90 & 3,29 & {$[1,1]$} \\
$\mathbf{2 0}$ & 118 & 62,01 & 3,10 & {$[1,1]$} \\
$\mathbf{3 0}$ & 177 & 91,45 & 3,05 & {$[1,1]$} \\
$\mathbf{4 0}$ & 237 & 120,45 & 3,01 & {$[1,1]$} \\
\hline
\end{tabular}

Tabela 5: Precondicionador Combinativo 1

da utilização de um precondicionador mais robusto para a resolução simultânea de vários lados direitos. As Tabelas 4, 5 e 6 mostram o desempenho do COMBESP em um problema trifásico com 8127 células. O critério de parada corresponde a um erro relativo de $10^{-5}$. Para problemas com 1, 10, 20, 30 e 40 lados direitos, realizamos várias experiências numéricas com $k_{r}$ e $k_{p}$ variando entre 1 e 7 , e apresentamos somente os valores de $\left[k_{r}, k_{p_{1}}\right]$ e $\left[k_{r}, k_{p_{2}}\right]$ que produziram os melhores tempos para os precondicionadores Combinativo 1 e 2, respectivamente. Como podíamos esperar, um precondicionador mais robusto tende a produzir melhores resultados quando há um número maior de lados direitos a resolver. Vemos ainda que o Combinativo 2 apresentou o melhor desempenho global para múltiplos lados direitos.

\begin{tabular}{c|cccc}
$\mathbf{n}$ & iterações & tempo total & tempo iteração & {$\left[k_{r}, k_{p_{2}}\right]$} \\
\hline \hline $\mathbf{1}$ & 13 & 6,26 & 6,26 & {$[1,1]$} \\
$\mathbf{1 0}$ & 98 & 34,83 & 3,48 & {$[1,3]$} \\
$\mathbf{2 0}$ & 163 & 61,35 & 3,07 & {$[1,4]$} \\
$\mathbf{3 0}$ & 296 & 94,86 & 3,16 & {$[1,3]$} \\
$\mathbf{4 0}$ & 327 & 117,05 & 2,93 & {$[1,5]$} \\
\hline
\end{tabular}

Tabela 6: Precondicionador Combinativo 2

Dos resultados obtidos, podemos afirmar que o COMBESP mostrou-se adequado ao propósito de por à disposição da Petrobrás S.A. um pacote computacional eficiente e de código aberto a alterações. A comparação com um pacote comercial revelou que ainda há espaço para aperfeiçoamentos. Estamos seguindo nesta direção. O objetivo de dotar a empresa de um instrumento eficiente para o estudo de ajuste histórico não foi atingido, visto que a técnica de sementes não funcionou. A resolução de diversos lados direitos é uma área de investigação muito importante, 
com várias aplicações, e ainda largamente incipiente.

Abstract. We describe a GMRES method of preconditioned generalized conjugate gradients to solve linear systems that appear in Oil Reservoir Simulation. Three preconditioners are proposed. Numerical results, and a comparison with a commercial simulator, are presented. In particular, an application to a parameter determination problem is discussed.

\section{Referências}

[1] O. Axelsson, "Iterative Solution Methods", Cambridge University Press, New York, 1994.

[2] K. Aziz e A. Settari, "Petroleum Reservoir Simulation", Applied Sci. Publ. , Londres, 1979.

[3] L.M. Carvalho, F. Dickstein, J.R.P. Rodrigues e R.W. Santos, "Relatório Interno Petrobrás", 2001.

[4] T. Chan e W.L. Wan, Analysis of projection methods for solving linear systems with multiple right-hand sides, SIAM J. Sci. Comput., 18 (1997), 1698-1721.

[5] G.H. Golub and C.F. Van Loan, "Matrix Computations", Third Edition, Johns Hopkins Univ. Press, Baltimore, 1996.

[6] C.T. Kelley, "Iterative Methods for Linear and Nonlinear Equations", SIAM, Philadelphia, 1995.

[7] B.N. Parlett, A new look at the Lanczos method for solving symmetric systems of linear equations, Linear Algebra Appl., 17 (1980), 323-346.

[8] Y. Saad, On the Lanczos method for solving symmetric systems with several right-hand sides, Math Comp., 48 (1987), 651-662.

[9] Y. Saad. GMRES: a generalized minimal residual algorithm for solving nonsymmetric linear systems, SIAM J. Sci. Stat. Comput., 7(1986), 856-869.

[10] Y. Saad. "Iterative Methods for Sparse Linear Sistems", second edition with corrections, 2000.

[11] V. Simoncini e E. Gallopoulos, An iterative method for nonsymmetric systems with multiple right-hand sides, SIAM J. Sci. Comput., 18 (1995), 917-933.

[12] C.F. Smith, A.F. Peterson e R. Mittra, A conjugate gradient algorithm for the treatment of multiple incident eletromagnetic fields, IEEE Trans. on Antennas and Propag., 37, No. 11 (1989), 1490-1493.

[13] H.A. Van der Vorst, An iterative solution method for solving $f(A) x=b$, using Krylov subspace information obtained for the symmetric positive matrix $A, J$. of Comput. and Appl. Math., 18 (1987), 249-263. 
[14] J. Wallis, "Incomplete Gaussian Elimination as a Preconditioning for Generalized Conjugate Gradient Acceleration", SPE, 12265, 1983.

[15] M.F. Wheeler, "Numerical Simulation in Oil Recovery", Springer-Verlag, Nova Iorque, 1988. 